Research Paper

\title{
Ratio of Autoantibodies of Tumor Suppressor AIMP2 and Its Oncogenic Variant Is Associated with Clinical Outcome in Lung Cancer
}

\author{
Ji Ye Jung1, Eun Young Kim¹, Arum Kim², Joon Chang1, Nam Hoon Kwon³, Youngji Moon³, Eun Joo Kang? \\ Jun Sik Sung3, Hyunbo Shim ${ }^{4}$, Sunghoon Kim ${ }^{3}, 5$, Yoon Soo Chang ${ }^{2 \bowtie}$ \\ 1. Division of Pulmonology, Department of Internal Medicine, Severance Hospital, Yonsei University College of Medicine, Seoul, Korea; \\ 2. Division of Pulmonology, Department of Internal Medicine, Gangnam Severance Hospital, Yonsei University College of Medicine, Seoul, Korea: \\ 3. Medicinal Bioconvergence Research Center, Seoul National University, Seoul, Korea; \\ 4. Department of Life Science and Pharmaceutical Science, Ewha Womans University, Seoul, Korea; \\ 5. WCU Department of Molecular Medicine and Biopharmaceutical Sciences, Seoul National University, Suwon, Korea. \\ $\triangle$ Corresponding author: Yoon Soo Chang, 8th Floor, Annex Building, 211 Eonju-ro, Gangnam-gu, Seoul 06273, Republic of Korea. Tel.: +82-2-2019-3309, Fax: \\ +82-2-3463-3882. E-mail address: yschang@yuhs.ac
}

( ( Ivyspring International Publisher. This is an open access article distributed under the terms of the Creative Commons Attribution (CC BY-NC) license (https://creativecommons.org/licenses/by-nc/4.0/). See http://ivyspring.com/terms for full terms and conditions.

Received: 2016.11.22; Accepted: 2017.03.06; Published: 2017.05.12

\begin{abstract}
Aminoacyl-tRNA synthetase-interacting multi-functional protein 2 (AIMP2) works as potent tumor suppressor, while its splicing variant lacking exon 2 (AIMP2-DX2) competes with AIMP2 for binding to target proteins and compromises its anti-tumor activity. Assuming that AIMP2 and its variant AIMP2-DX2 could be released out to human sera in pathological condition, we investigated the diagnostic and prognostic usefulness of autoantibodies against AIMP2 and AIMP2-DX2 by measuring their serum levels in 80 normal and lung cancer samples that were matched in age, gender and smoking status. The area under the curve of AIMP2-DX2, AIMP2, and AIMP2-DX2/AIMP2 autoantibody ratio was low $(0.416,0.579$, and 0.357 , respectively), suggesting limited diagnostic value. A total of 165 lung cancer patients were classified into low and high AIMP2-DX2, AIMP2, and AIMP2-DX2/AIMP2 based on the median expression of each parameter. The high AIMP2-DX2 group was older and had larger tumors $(>3 \mathrm{~cm})$ than the low AIMP2-DX2 group. The high AIMP2-DX2/AIMP2 group had higher CYFRA-21 levels and significantly shorter overall survival than the low AIMP2-DX2/AIMP2 group (18.6 vs. 48.9 months, $P=0.021$, Log Rank Test). Taken together, autoantibodies against AIMP2-DX2 and AIMP2 are detectable in the human blood and the increased ratio of AIMP2-DX2/AIMP2 is related to poor clinical outcome of lung cancer.
\end{abstract}

Key words: Aminoacyl t-RNA synthetase (ARS), Aminoacyl t-RNA synthetase-interacting multi-functional protein 2 (AIMP2), Aminoacyl t-RNA synthetase-interacting multi-functional protein 2-exon 2 deletion (AIMP2-DX2), Autoantibody, Lung cancer.

\section{Introduction}

Lung cancer is the second most common malignancy, accounting for more than one-quarter of all cancer deaths in the United States.[1] Despite the unremitting efforts toward biomarker discovery and the development of targeted therapy and immunotherapy, lung cancer is by far the leading cause of cancer death with poor prognosis. Development of effective tools for early diagnosis and new therapeutic strategies based on lung cancer biology are urgently needed to improve its clinical outcome.

Aminoacyl-tRNA synthetase-interacting multifunctional proteins (AIMPs) are scaffolding proteins for the assembly of the macromolecular tRNA synthetase complex. Among the 3 types of AIMPs, AIMP2 (also known as p38 and JTV1) possesses 
anti-proliferative and cell death-promoting activity through multiple pathways such as tumor-derived growth factor (TGF)- $\beta$, p53, tumor necrosis factor (TNF)-a signaling and Wnt signaling.[2-8] Mice lacking AIMP2 die neonatally due to respiratory failure resulting from uncontrolled proliferation of lung epithelial cells. AIMP2 heterozygous mice (with reduced expression of AIMP2) show a higher susceptibility to tumorigenesis.[5] These findings indicate that AIMP2 is a haploinsufficient tumor suppressor with a unique working mechanism.[2]

A variant of AIMP2 in which exon 2 is deleted by alternative splicing, AIMP2-DX2, has been detected in cancer cell lines and tissues.[9] AIMP2-DX2 competes with AIMP2 for the binding to target proteins (namely FUSE-binding protein [FBP], TNF receptor-associated factor 2 [TRAF2], and p53), consequently inhibiting the tumor suppressive activity of AIMP2. Increased ratio of AIMP2-DX2 versus AIMP2 showed positive correlation to poor clinical outcome of lung cancer, as well as chemoresistance of ovarian cancer.[2, 9] The synthetic compound, BC-DXI01suppressed the AIMP2-DX2 mRNA transcript, leading to the inhibition of the AIMP2-DX2 activity and tumor suppression.[10] Taken together, these data suggest that AIMP2-DX2 is an attractive candidate for cancer diagnosis and therapy.

In this study, AIMP2-DX2 expression was examined in lung cancer using Kras and Kras:p53 fl/fl mouse lung cancer models and human lung cancer tissues. Moreover, the presence of autoantibodies against AIMP2-DX2 and AIMP2 in the blood was investigated by enzyme-linked immunosorbent assay (ELISA). We sought to find the diagnostic and prognostic usefulness of these autoantibodies in lung cancer patients.

\section{Materials and Methods}

\section{Tissue samples}

Mouse tissues were obtained from wild-type C57BL/6, LSL-Kras G12D, and LSL-Kras G12D:p53 fl/fl mice. For mouse lung cancer tissues, LSL-Kras G12D and LSL-Kras G12D:p53 fl/fl mice (http://mouse.ncifcrf.gov/) inhaled $5 \times 10^{7} \mathrm{PFU}$ AdCre particles at 8 weeks of age and sacrificed at 8 and 24 weeks after inhalation, respectively. This animal study was approved by our Institutional Animal Care and Use Committee (2014-0229-1), following the guidelines of the American Association for the Assessment and Accreditation of Laboratory Animal Care. A total of 275 formalin-fixed, paraffin-embedded human lung cancer tissues, and 12 pairs of lung cancer and adjacent normal-appearing lung tissue lysates were obtained from tissue archives of affiliated hospitals of Yonsei University College of Medicine. The use of clinical samples was approved by the Institutional Review Board of the Severance Hospital (\#4-2013-0556) and Gangnam Severance Hospital (\#3-2014-0838).

\section{Serum samples}

The serum samples from lung cancer patients and control subjects were obtained from those who had presented to Severance Hospital and Gangnam Severance Hospital between 2011 September and 2015 January. Lung cancer cases were confirmed pathologically and did not present with any other types of cancer. Control cases were 1:1 age-, genderand smoking status-matched and did not present with any type of cancer. A total of 165 samples from lung cancer patients were included. Informed consent was obtained from all study subjects, and all protocols were approved by the Institutional Review Board of the Severance Hospital (\#4-2013-0556) and Gangnam Severance Hospital (\#3-2014-0838). The study was carried out in accordance with the Declaration of Helsinki and Korean GCP guidelines.

\section{Immunoblotting}

Tissues were harvested using $2 \times$ LSB lysis buffer containing protease and phosphatase inhibitors (GenDepo, Korea) on ice. After homogenization and sonication, $30-50 \mathrm{mg}$ of lysates was separated by gel electrophoresis on $7.5-12 \%$ polyacrylamide gels and transferred onto nitrocellulose membranes (Bio-Rad Laboratories, Inc., Richmond, CA, USA). The expression level of each protein was measured using Image J (http://rsbweb.nih.gov/ij/) and quantified relative to that of $\beta$-actin.

\section{Immunohistochemistry (IHC)}

Expression of AIMP2-DX2 in mouse lung tissue and human lung cancer tissue was analyzed by IHC using the LABS ${ }^{\circledR 2}$ System (Dako, Carpinteria, CA, USA) according to the manufacturer's instructions. Briefly, sections were deparaffinized, rehydrated, immersed in $\mathrm{H}_{2} \mathrm{O}_{2}$ methanol solution, and then incubated overnight with primary antibodies against AIMP2-DX2 obtained by rabbit immunization at a 1:2,000 dilution. Sections were incubated for 10 minutes with biotinylated linker and processed using avidin/biotin IHC techniques. 3,3

-Diaminobenzidine (DAB) was used as a chromogen in conjunction with the Liquid DAB Substrate kit (Novacastra, UK). Expression of AIMP2-DX2 was evaluated using a scoring system that takes into account the product of staining intensity and percentage of positive cells. Staining intensity was classified as $0,1,2$, and 3 and frequency was classified 
as $0(<10 \%), 1(10-50 \%), 2(51-80 \%)$, and $3(>80 \%)$ (Fig. S1). The cases were classified into 2 groups according to the AIMP2-DX2 expression in the cytoplasm and nucleus. Cases with cytoplasmic AIMP2-DX2 expression intensity and frequency equal to or greater than 4 were defined as having high expression; those with 3 or less were defined as having low expression. Cases with nuclear AIMP2-DX2 expression equal to or greater than 1 were defined as having high expression; those with 0 were defined as having low expression.

\section{Measurement of serum autoantibodies against AIMP2 and AIMP2-DX2}

The 96-well plates were coated with either His-tagged AIMP2-DX2 or His-tagged AIMP2 protein (Neomix Inc, Korea) overnight at $4^{\circ} \mathrm{C}$, and then blocked with $1 \%$ bovine serum albumin for 1 hour at room temperature. After adding 1:500 diluted serum, 1:10,000 dilution anti-human immunoglobulin $G$ was added and incubated for another 1 hour at room temperature. The enzyme activity was visualized by adding 3,3', 5,5'-tetramethylbenzidine substrate (TMB; Thermo Fisher Scientific) for 10 minutes and the reaction was stopped by $2 \mathrm{~N} \quad \mathrm{H}_{2} \mathrm{SO}_{4}$. The absorbance was measured at $450 \mathrm{~nm}$ was measured. The intra-assay variation of AIMP2-DX2 and AIMP2 was $8.7 \% \quad(6.8-11.5 \%)$ and $6.3 \%$ (1.6-10.4\%), respectively. The inter-assay (plate-to-plate) variation was $15.9 \%$ (13.7-17.9\%) and $12.2 \%$ (8.9-16.1\%), respectively. Lung cancer patients were classified into 2 groups, high and low, on the basis of the median values of AIMP2-DX2, AIMP2, and AIMP2-DX2/ AIMP2 ratio.

\section{Statistical analysis}

Significant differences in clinical characteristics according to the expression of AIMP2-DX2 by IHC in lung tissues, and AIMP2-DX2, AIMP2, and AIMP2-DX2/AIMP2 autoantibody ratio were analyzed using the $\chi^{2}$-test, Fisher's exact test, and independent 2-sample t-test. Predictive factors for overall survival (OS) and progression/recurrence-free survival (PFS) were calculated using the Kaplan-Meier method and Cox proportional hazards model. All tests of significance were 2-tailed. A $P$-value less than 0.05 was interpreted as statistical significance. All analyses were performed using SPSS version 20 (SPSS Inc., Chicago, IL, USA).

\section{Results}

\section{AIMP2-DX2 is overexpressed in lung cancer tissue}

To explore AIMP2-DX2 as a biomarker of lung cancer, its expression was first evaluated in the non-neoplastic lung tissue from 8-week-old wild-type C57BL/6 mice using the antibody specific to AIMP2-DX2 (Fig. S2A). Cells comprising alveolar structure did not express AIMP2-DX2, while bronchial epithelial cells showed weak expression. To evaluate if AIMP2-DX2 expression is specific to lung cancer, its expression was evaluated in lung cancer tissues. Lung cancer-specific AIMP2-DX2 overexpression was observed in Kras-LSL G12D and Kras-LSL G12D:p53fl/fl murine lung cancer models induced by AdCre inhalation (Fig. S2B). The expression of AIMP2-DX2 was also evaluated in normal-appearing human tissues adjacent to lung cancer tissues (Fig. S2C). AIMP2-DX2 was not expressed in type I and type II pneumocytes and was weakly expressed in the epithelial cells of the bronchioles and bronchi. In human lung cancer tissues, AIMP2-DX2 was strongly expressed mainly in the cytoplasm of adenocarcinoma, squamous cell carcinoma, and small cell lung cancer, and weakly expressed in large cell carcinoma (Fig. S2D). Lung cancer-specific AIMP2-DX2 overexpression was further evaluated by immunoblotting. In mouse lung tissue, AIMP2-DX2 expression was stronger in lung cancer than in normal lungs (Fig. S2E). Among 12 pairs of human lung cancer tissue and adjacent normal-appearing lung tissue, 7 pairs (58.3\%) showed stronger overexpression of AIMP2-DX2 in lung cancer tissue (Fig. S2F). To further validate its clinical implication in lung cancer, its expression was assessed in 275 formalin-fixed, paraffin-embedded lung cancer tissues. The baseline characteristics of the 275 patients are shown in Table S1. The distribution of cytoplasmic and nuclear AIMP2-DX2 expression by IHC is shown in Fig. S2G and S2H. Among the 275 patients, $270(98.2 \%)$ showed positive cytoplasmic AIMP2-DX2 expression while 91 (33.1\%) showed positive nuclear AIMP2-DX2 expression. The clinical and pathological characteristics of patients with high and low cytoplasmic/nuclear expression are shown in Table S2. High cytoplasmic $(39.6 \%$ vs. $28.6 \%, P=$ $0.055)$ and nuclear AIMP2-DX2 expression $(42.9 \%$ vs. $30.4 \%, P=0.042)$ was more frequent in advanced stages (III-IV) than in earlier stages (I-II). High cytoplasmic $(58.2 \%$ vs. $40.2 \%, P=0.003)$ and nuclear AIMP2-DX2 expression ( $58.4 \%$ vs. $45.8 \%, P=0.052$ ) was more frequent in adenocarcinoma than in squamous cell carcinoma. However, the level of AIMP2-DX2 expression was not related with clinical outcome, with no significant differences in OS and PFS (Fig. S3).

\section{Autoantibodies against AIMP2-DX2 and AIMP2 are detectable in the human blood}

The baseline characteristics of age, gender, and 
smoking habits were not different between lung cancer $(n=80)$ and control patients $(n=80)$ (Table 1$)$. Interestingly, autoantibodies against AIMP2-DX2 and AIMP2 were detectable in the serum (Fig. 1A). When their diagnostic usefulness was evaluated by receiver operating characteristic curve analysis, the area under the curve of AIMP2-DX2, AIMP2, and AIMP2-DX2/AIMP2 autoantibody ratio was 0.416, 0.579 , and 0.357 , respectively, suggesting that their diagnostic value in lung cancer is limited (Fig. 1B).
Table 1. Baseline characteristics of control and lung cancer patients ${ }^{\mathrm{a}}$

\begin{tabular}{llll}
\hline Baseline characteristics & Control $(\mathrm{n}=80)$ & $\begin{array}{l}\text { Lung cancer } \\
(\mathrm{n}=80)\end{array}$ & $P$-value \\
\hline Age & $68.2 \pm 12.5$ & $68.5 \pm 9.3$ & 0.869 \\
Gender, male & $63(78.8)$ & $61(76.3)$ & 0.705 \\
$\begin{array}{l}\text { Smoking status, ever-smoker } \\
\text { Pack years (including }\end{array}$ & $32.6 \pm 28.8$ & $35.5 \pm 30.5$ & 0.549 \\
none-smokers) & & & 1.000 \\
\hline
\end{tabular}

a Data are presented as numbers (percentages) unless otherwise indicated.

Plus-minus values are means \pm standard deviation.

A

Control

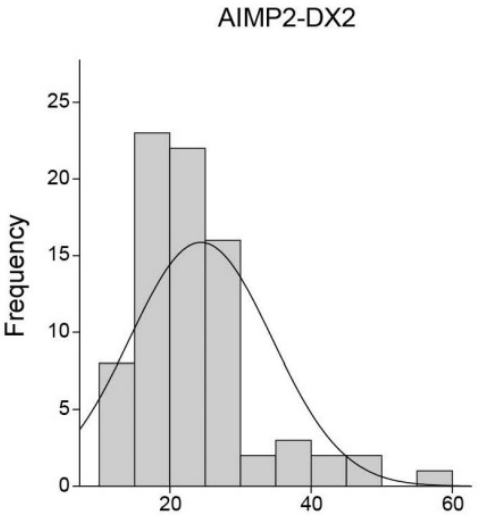

$(\mathrm{AU})$

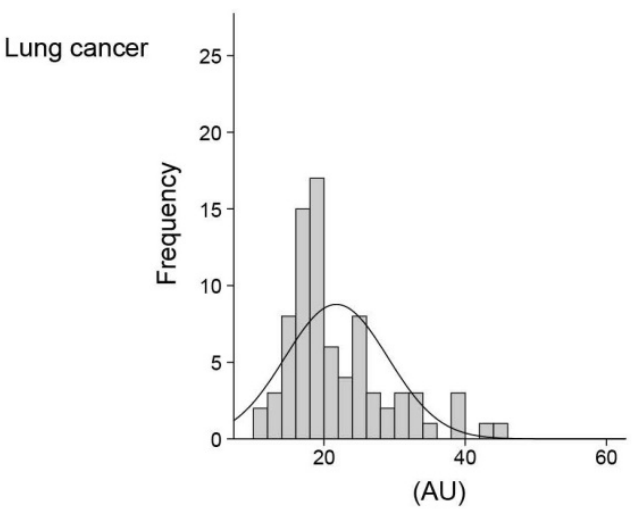

B

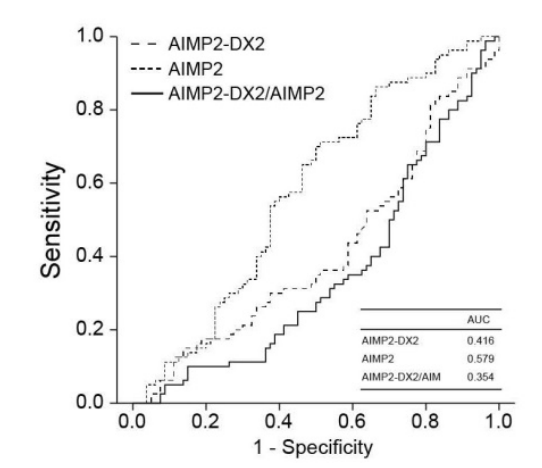

AIMP2
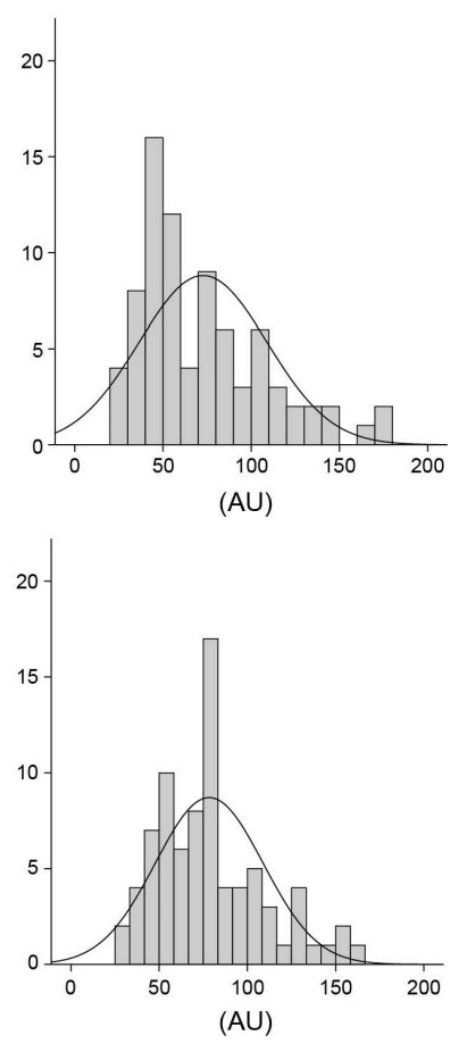

AIMP2-DX2/AIMP2 ratio
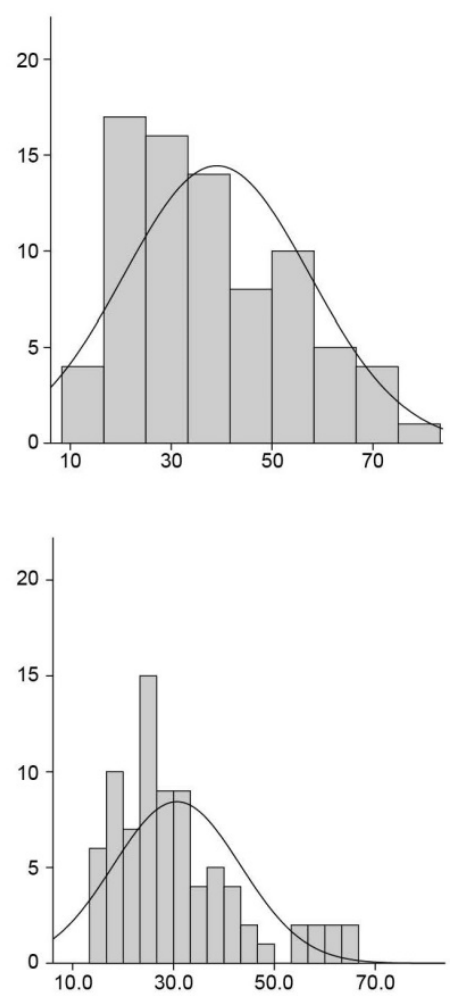

Figure 1. Histogram for distribution of AIMP2-DX2, AIMP2, and AIMP2-DX2/AIMP2 autoantibody ratio and receiver operating characteristic curve analysis of the diagnostic value for lung cancer. (A) Histogram for distribution of AIMP2-DX2, AIMP2, and AIMP2-DX2/AIMP2 autoantibody ratio in control and lung cancer patients. (B) Receiver operating characteristic curve of AIMP2, AIMP2-DX2, and AIMP2-DX2/AIMP2 ratio for the diagnosis of lung cancer. AIMP2: aminoacyl-tRNA synthetase-interacting multi-functional protein 2, AIMP2-DX2: aminoacyl-tRNA synthetase-interacting multi-functional protein 2-exon 2 deletion 


\section{AIMP2-DX2 autoantibody levels are related with tumor size}

AIMP2-DX2 and AIMP2 autoantibody levels were measured in 165 lung cancer patients, and the AIMP2-DX2/AIMP2 autoantibody ratio was calculated. The baseline characteristics of the included patients are shown in Table 2. Mean age was 65 years and $64.8 \%$ of the patients were male. About one-third of the patients were never-smokers. According to histologic classification, adenocarcinoma was the most common type $(62.4 \%)$, followed by squamous cell carcinoma (25.5\%). AIMP2-DX2, AIMP2, and AIMP2-DX2/AIMP2 autoantibody ratio levels were $21.6 \pm 9.2,76.7 \pm 33.8$, and $31.7 \pm 13.8$ AU, respectively. The baseline characteristics of low and high AIMP2-DX2, AIMP2, and AIMP2-DX2/AIMP2 ratio groups are shown in Table 3 . There was no statistical difference in gender, smoking, histologic diagnosis, and stage between high and low group of AIMP2-DX2, AIMP2, and AIMP2-DX2/AIMP2 ratio. However, the high AIMP2-DX2 autoantibody group was older (66.8 \pm 8.4 vs. $63.8 \pm 10.1$ years, $P=0.040)$ and had larger tumors $(>3 \mathrm{~cm})(26.9 \%$ vs. $12.7 \%, P=$ $0.045)$ than the low group. Elevated CYFRA-21 levels were more frequently observed in the high AIMP2-DX2/AIMP2 autoantibody ratio group than in the low group $(58.8 \%$ vs. $41.2 \%, P=0.037)$, but the frequency of elevated carcinoembryonic antigen (CEA) was similar between the groups. Moreover, the mortality rate was higher in the high AIMP2-DX2/AIMP2 autoantibody ratio group than in the low group $(58.5 \%$ vs. $42.0 \%, P=0.034)$.
Table 2. Characteristics of lung cancer patients ${ }^{\mathrm{a}}$

\begin{tabular}{|c|c|}
\hline Characteristics & $\mathrm{n}=165$ \\
\hline Age, yr & $65.3 \pm 9.4$ \\
\hline Sex, male & $107(64.8)$ \\
\hline \multicolumn{2}{|l|}{ Smoking } \\
\hline Current & $58(35.2)$ \\
\hline Former & $50(30.3)$ \\
\hline Never & $57(34.5)$ \\
\hline \multicolumn{2}{|l|}{ Histology } \\
\hline Adenocarcinoma & $103(62.4)$ \\
\hline Squamous cell carcinoma & $42(25.5)$ \\
\hline Small cell carcinoma & $16(9.7)$ \\
\hline Large cell carcinoma & $4(2.4)$ \\
\hline Size $>3 \mathrm{~cm}^{\mathrm{b}}$ & $23(18.5)$ \\
\hline \multicolumn{2}{|l|}{ Stage $(n=149)^{c}$} \\
\hline 1 & $47(31.5)$ \\
\hline 2 & $8(5.4)$ \\
\hline 3 & $36(24.2)$ \\
\hline 4 & $58(38.9)$ \\
\hline \multicolumn{2}{|l|}{ Tumor markers } \\
\hline Elevated CEAd & $74(49.3)$ \\
\hline Elevated CYFRA 21-1e & $68(47.6)$ \\
\hline \multicolumn{2}{|l|}{ Novel markers, AU } \\
\hline AIMP2-DX2 & $21.6 \pm 9.2$ \\
\hline AIMP2 & $76.7 \pm 33.8$ \\
\hline AIMP2-DX2/AIMP2 & $31.7 \pm 13.8$ \\
\hline Mortality $(n=163)^{f}$ & $82(50.3)$ \\
\hline \multicolumn{2}{|c|}{ a Data are presented as numbers (percentages) unless otherwise indicated. } \\
\hline \multicolumn{2}{|l|}{${ }^{\mathrm{b}} \mathrm{n}=124$} \\
\hline \multicolumn{2}{|c|}{ c Small cell carcinoma was excluded } \\
\hline \multicolumn{2}{|l|}{ e $>3.3 \mathrm{ng} / \mathrm{mL}, \mathrm{n}=143$} \\
\hline \multicolumn{2}{|c|}{ f Survival of two patients are unknown } \\
\hline \multicolumn{2}{|c|}{$\begin{array}{l}\text { CEA: carcinoembryonic antigen, AIMP2: aminoacyl-tRNA synthetase interacting } \\
\text { multi-functional protein 2, AIMP2-DX2: aminoacyl-tRNA synthetase interacting } \\
\text { multi-functional protein 2-exon } 2 \text { deletion (AIMP2-DX2) }\end{array}$} \\
\hline
\end{tabular}

Table 3. Baseline characteristics of low and high AIMP2-DX2, AIMP2, and AIMP2-DX2/AIMP2 autoantibody ratio groups

\begin{tabular}{|c|c|c|c|c|c|c|c|c|c|}
\hline \multirow[t]{2}{*}{ Characteristics } & \multicolumn{3}{|c|}{ AIMP2-DX2b } & \multicolumn{3}{|l|}{ AIMP2b } & \multicolumn{3}{|c|}{ AIMP2-DX2/AIMP2b } \\
\hline & $\begin{array}{l}\text { Low } \\
(n=82)\end{array}$ & $\begin{array}{l}\text { High } \\
(\mathrm{n}=83)\end{array}$ & $P$-value & $\begin{array}{l}\text { Low } \\
(n=82)\end{array}$ & $\begin{array}{l}\begin{array}{l}\text { High } \\
(\mathrm{n}=83)\end{array}\end{array}$ & $P$-value & $\begin{array}{l}\text { Low } \\
(n=82)\end{array}$ & $\begin{array}{l}\text { High } \\
(\mathrm{n}=83)\end{array}$ & $P$-value \\
\hline Age, yr & $63.8 \pm 10.1$ & $66.8 \pm 8.4$ & 0.040 & $64.1 \pm 9.4$ & $66.5 \pm 9.3$ & 0.113 & $64.7 \pm 9.5$ & $65.9 \pm 9.2$ & 0.443 \\
\hline Sex, male & $52(63.4)$ & $55(67.1)$ & 0.623 & $51(62.2)$ & $56(67.5)$ & 0.478 & $54(65.9)$ & $53(63.9)$ & 0.788 \\
\hline \multicolumn{10}{|l|}{ Smoking } \\
\hline Current & $32(39.0)$ & $26(31.7)$ & 0.603 & $27(32.9)$ & $31(37.3)$ & 0.484 & $29(35.4)$ & $29(34.9)$ & 0.686 \\
\hline Former & $23(28.0)$ & $27(32.9)$ & & $23(28.0)$ & $27(32.5)$ & & $27(32.9)$ & $23(27.7)$ & \\
\hline Never & $27(32.9)$ & $29(35.4)$ & & $32(39.0)$ & $25(30.1)$ & & $26(31.7)$ & $31(37.3)$ & \\
\hline \multicolumn{10}{|l|}{ Histology } \\
\hline Adenocarcinoma & $55(67.1)$ & $48(57.3)$ & 0.478 & $53(64.6)$ & $50(60.2)$ & 0.216 & $53(64.6)$ & $50(60.2)$ & 0.580 \\
\hline Squamous cell carcinoma & $18(22.0)$ & $24(29.2)$ & & $16(19.5)$ & $26(31.3)$ & & $20(24.4)$ & $22(26.5)$ & \\
\hline Small cell carcinoma & $8(9.7)$ & $8(9.8)$ & & $10(12.2)$ & $6(7.2)$ & & $7(8.5)$ & $9(10.8)$ & \\
\hline Large cell carcinoma & $1(1.2)$ & $3(3.7)$ & & $3(3.7)$ & $1(1.2)$ & & $2(2.4)$ & $2(2.4)$ & \\
\hline Size $>3 \mathrm{~cm}$ & $9(12.7)$ & $14(26.9)$ & 0.045 & $12(18.2)$ & $11(19.0)$ & 0.911 & $13(20.0)$ & $10(16.9)$ & 0.662 \\
\hline \multicolumn{10}{|l|}{ Stage $(n=149)$} \\
\hline I-II & $34(41.5)$ & $27(33.3)$ & 0.284 & $28(34.1)$ & $34(41.5)$ & 0.334 & $36(44.4)$ & $45(55.6)$ & 0.083 \\
\hline III-IV & $48(58.5)$ & $54(66.7)$ & & $54(65.9)$ & $48(58.5)$ & & $26(31.3)$ & $57(68.7)$ & \\
\hline \multicolumn{10}{|l|}{ Tumor markers } \\
\hline Elevated CEAc & $42(56.8)$ & $32(43.2)$ & 0.119 & $40(54.1)$ & $34(45.9)$ & 0.193 & $35(47.3)$ & $39(52.7)$ & 0.514 \\
\hline Elevated CYFRA-21d & $38(55.9)$ & $30(44.1)$ & 0.307 & $37(54.4)$ & $31(45.6)$ & 0.278 & $28(41.2)$ & $40(58.8)$ & 0.037 \\
\hline Mortality & $37(45.1)$ & $45(56.3)$ & 0.157 & $42(51.9)$ & $40(48.8)$ & 0.695 & $34(42.0)$ & $48(58.5)$ & 0.034 \\
\hline $\begin{array}{l}\text { b High and low groups were cl } \\
\text { c }>5.0 \mathrm{ng} / \mathrm{mL} ; \mathrm{n}=75 \text { for low gr } \\
\text { d }>3.3 \mathrm{ng} / \mathrm{mL} ; \mathrm{n}=72 \text { for low } \mathrm{gr}\end{array}$ & $\begin{array}{l}5 \text { for high } g \\
71 \text { for high }\end{array}$ & ald & $12-1$ & 112 & $X 2 / \mathrm{A}$ & . & & & \\
\hline
\end{tabular}




\section{Elevated AIMP2-DX2/AIMP2 autoantibody ratio is related with poor clinical outcome in lung cancer}

To evaluate the prognostic value of AIMP2-DX2, AIMP2, and AIMP2-DX2/AIMP2 autoantibody ratio in lung cancer, OS and PFS were compared using Kaplan-Meier estimators (Fig. 2). There were no significant differences in PFS and OS between low and high AIMP2-DX2 and AIMP2 autoantibody groups. However, the patients with high AIMP2-DX2/AIMP2 autoantibody ratio showed significantly shorter OS (18.4 months [95\% confidence interval (CI) 15.1-21.7] vs. 48.3 months [95\% CI 14.6-81.9], $P=0.021$ ). To evaluate the hypothesis that high AIMP2-DX2/AIMP2 ratio is an independent prognostic factor in lung cancer, univariate and multivariate analyses were performed using a Cox regression hazard model (Table 4). Univariate analysis revealed that age, female, ever-smoker, advanced stages (III-IV), elevated CEA, elevated CYFRA-21, and high AIMP2-DX2/AIMP2 autoantibody ratio were significant predictors for poor OS while adenocarcinoma histology was a significant predictor for better OS. Multivariate analysis showed that advanced stages (III-IV) (HR = 6.66, 95\% CI 3.20-13.89) and high AIMP2-DX2/AIMP2 autoantibody ratio $(\mathrm{HR}=1.83$, 95\% CI 1.11-3.00) were significant independent factors for poor OS while adenocarcinoma histology $(\mathrm{HR}=0.42,95 \%$ CI 0.23-0.76) was a significant predictor for better OS.
A
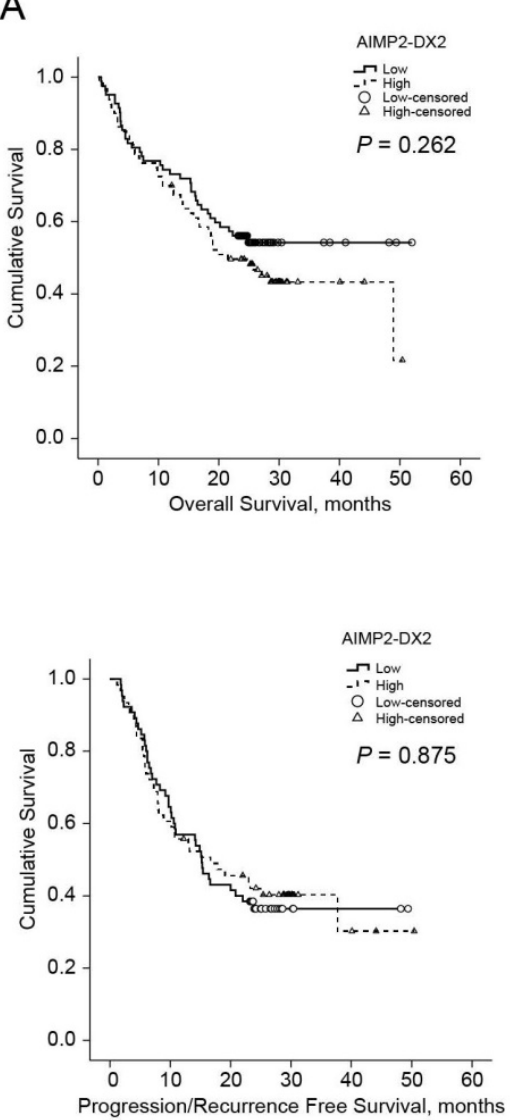

B
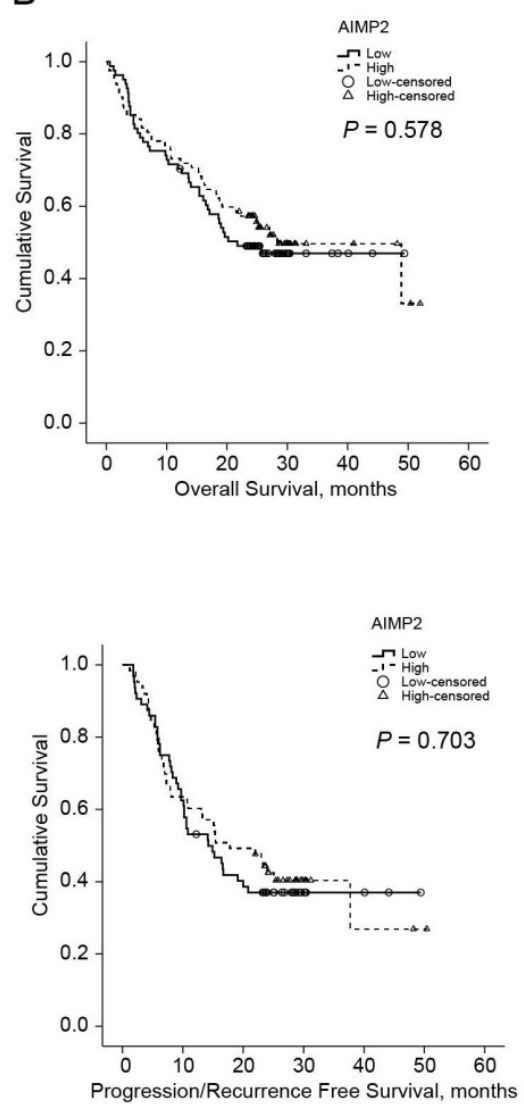

C
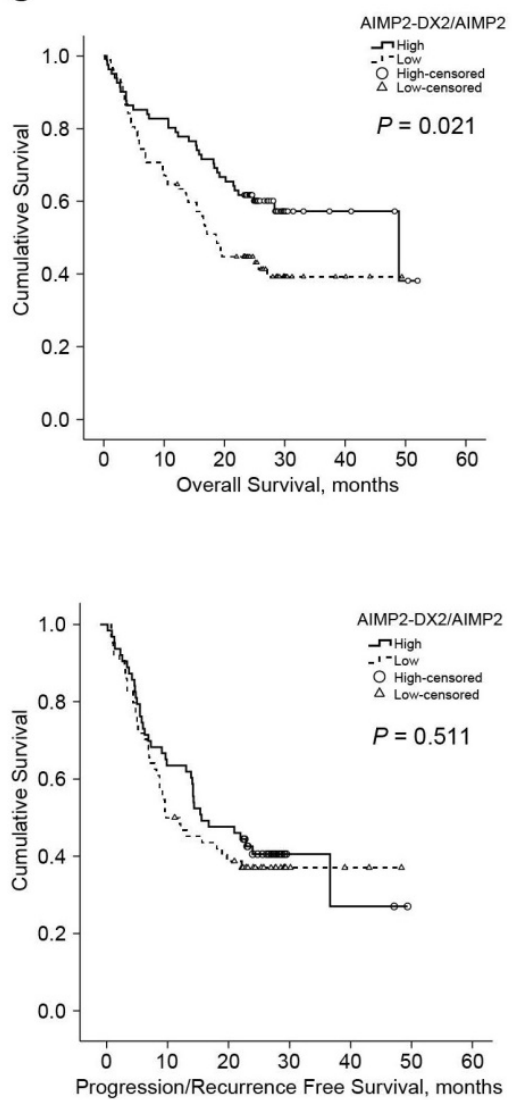

\begin{tabular}{ccccccc}
\hline \multirow{2}{*}{ Median } & \multicolumn{2}{c}{ AIMP2-DX2 } & \multicolumn{2}{c}{ AIMP2 } & \multicolumn{2}{c}{ AlMP2-DX2 / AIMP2 } \\
& Low & High & Low & High & Low & High \\
\hline OS & - & 21.1 & 21.4 & 27.9 & 48.3 & 18.4 \\
months $(95 \% \mathrm{Cl})$ & & $(13.0-29.5)$ & $(-)$ & $(15.6-40.2)$ & $(14.6-81.9)$ & $(15.1-21.7)$ \\
PFS & 14.9 & 15.0 & 13.9 & 17.5 & 16.2 & 10.7 \\
months $(95 \% \mathrm{Cl})$ & $(10.1-19.7)$ & $(6.2-23.8)$ & $(8.7-19.1)$ & $(8.1-26.9)$ & $(9.7-22.7)$ & $(6.6-14.8)$ \\
\hline
\end{tabular}

Figure 2. Impact of AIMP2-DX2, AIMP2, and AIMP2-DX2/AIMP2 ratio on overall survival and progression/recurrence-free survival in lung cancer patients. (A) AIMP2-DX2, (B) AIMP2, and (C) AIMP2-DX2/AIMP2 ratio. High and low groups were classified on the basis of the median values of AIMP2-DX2, AIMP2, and AIMP2-DX2/AIMP2 ratio. P-values were obtained by the log-rank test. AIMP2: aminoacyl-tRNA synthetase-interacting multi-functional protein 2, AIMP2-DX2: aminoacyl-tRNA synthetase-interacting multi-functional protein 2-exon 2 deletion, OS: overall survival, PFS: progression/recurrence-free survival 
Table 4. Univariate and multivariate Cox-regression analysis of overall survival

\begin{tabular}{|c|c|c|c|c|c|c|}
\hline \multirow[t]{2}{*}{ Characteristics } & \multicolumn{3}{|c|}{ Univariate Analysis } & \multicolumn{3}{|c|}{ Multivariate Analysis } \\
\hline & $\overline{\mathrm{HR}}$ & $P$-value & $95 \% \mathrm{CI}$ & HR & $P$-value & $95 \% \mathrm{CI}$ \\
\hline Age, yr & 1.03 & 0.022 & $1.00-1.06$ & 1.02 & 0.201 & $0.99-1.05$ \\
\hline Sex, female & 0.53 & 0.011 & $0.32-0.86$ & 0.76 & 0.655 & $0.22-2.58$ \\
\hline Smoking, ever-smoker & 1.71 & 0.029 & $1.06-2.77$ & 1.12 & 0.858 & $0.33-3.77$ \\
\hline \multicolumn{7}{|l|}{ Histology } \\
\hline Adenocarcinoma & 0.54 & 0.006 & $0.35-0.84$ & 0.42 & 0.004 & $0.23-0.76$ \\
\hline Squamous cell carcinoma & 1.51 & 0.084 & $0.95-2.42$ & & & \\
\hline Size $>3 \mathrm{~cm}$ & 0.56 & 0.180 & $0.24-1.31$ & & & \\
\hline Stage, stage III-IV vs. I-II & 6.71 & $<0.001$ & $3.45-13.05$ & 6.66 & $<0.001$ & $3.20-13.88$ \\
\hline \multicolumn{7}{|l|}{ Tumor markers } \\
\hline Elevated CEA ${ }^{a}$ & 1.92 & 0.007 & $1.20-3.07$ & 1.44 & 0.176 & $0.85-2.42$ \\
\hline Elevated CYFRA-21 b & 2.98 & $<0.001$ & $1.82-4.86$ & 1.40 & 0.231 & $0.81-2.41$ \\
\hline \multicolumn{7}{|l|}{ Novel markers ${ }^{c}$} \\
\hline High group of AIMP2-DX2 & 1.28 & 0.263 & $0.83-1.98$ & & & \\
\hline High group of AIMP2 & 0.88 & 0.579 & $0.57-1.37$ & & & \\
\hline High group of AIMP2-DX2/AIMP2 & 1.67 & 0.023 & $1.07-2.60$ & 1.83 & 0.018 & $1.11-3.00$ \\
\hline
\end{tabular}

a $>5.0 \mathrm{ng} / \mathrm{mL}$

b $>3.3 \mathrm{ng} / \mathrm{mL}$

c High group was classified on the basis of median value of AIMP2-DX2, AIMP2, and AIMP2-DX2/AIMP2 ratio, respectively

AIMP2: aminoacyl-tRNA synthetase interacting multi-functional protein 2, AIMP2-DX2: aminoacyl-tRNA synthetase interacting multi-functional protein 2-exon 2 deletion (AIMP2-DX2), HR: hazard ratio, CI: confidence interval

\section{Discussion}

This study investigated the clinical implications of AIMP2-DX2 tissue expression and serum autoantibodies in lung cancer patients. Although AIMP2-DX2 was frequently overexpressed in lung cancer tissues, and associated with advanced disease stages and adenocarcinoma, diagnostic usefulness of AIMP2-DX2 autoantibody was limited. Autoantibodies against AIMP2-DX2 and AIMP2 exist at detectable level in human blood and increased AIMP2-DX2/AIMP2 autoantibody ratio was an independent prognostic factor for poor OS in patients with lung cancer.

Although several reports have suggested the tumor promoting role of AIMP2-DX2, a potential of AIMP2-DX2 as a biomarker for cancer diagnosis, treatment, and prognosis prediction is limited.[2-7] In this study, we used immunohistological analysis using AIMP2-DX2-specific antibody to directly monitor AIMP2-DX2 at protein level rather than its transcript level and found its frequent increase $(54.2 \%)$ in NSCLC tissues.

There are pressing needs for the development of blood biomarkers because it is convenient and less invasive. There are a few biomarkers for the diagnosis and prognosis prediction in lung cancer and their usefulness is hampered because of low diagnostic and prognostic value.[11-13] Although it was unclear whether the soluble and secretory form of AIMP2-DX2 would exist in the blood, its autoantibody was detectable in the blood and showed the correlation with serum CYFRA 21-1 $(\gamma=0.133, P=$ 0.056; data not shown), a soluble fragment of cytokeratin 19 filaments.[14, 15]

The clinical implication of AIMP2, AIMP2-DX2 autoantibody and AIMP2-DX2/AIMP2 autoantibody ratio was investigated for the first time in this study from lung cancer patients. AIMP2-DX2/AIMP2 autoantibody ratio was suggested as a potential prognostic biomarker in lung cancer patients while AIMP2-DX2 autoantibody alone was not. The competitive interaction between AIMP2-DX2 and AIMP2 for binding to the target proteins could be related to the prognostic value of AIMP2-DX2/ AIMP2 ratio.

Although this study presented a potential of AIMP2-DX2 as a potential biomarker in lung cancer, there are a few limitations to be addressed. First, the diagnostic and prognostic value of AIMP2-DX2 as determined by IHC and autoantibody levels needs to be compared in the specimens obtained from the same subjects at the same time point. The relationship between AIMP2-DX2 in the tissue and in the blood is important for further clarifying the role of AIMP2-DX2 in cancer formation and progression. Second, development of direct measurement of AIMP2-DX2 and AIMP2 in the blood would be also needed to overcome the limitation of autoantibody analysis. Future studies should compare the secretory form of AIMP2-DX2/AIMP2 and autoantibodies against AIMP2-DX2/AIMP2 as suitable biomarkers in lung cancer. In conclusion, autoantibodies against AIMP2-DX2 and AIMP2 were detectable in blood. The increased ratio of AIMP2-DX2/AIMP2 autoantibodies was related to the poor clinical outcome of lung cancer patients, suggesting its potential as a prognostic marker in lung cancer.

\section{Supplementary Material}

Supplementary figures and tables.

http://www.jcancer.org/v08p1347s1.pdf 


\section{Abbreviations}

AIMP2: aminoacyl t-RNA synthetase-interacting multi-functional protein 2; AIMP2-DX2: aminoacyl t-RNA synthetase-interacting multi-functional protein 2-exon 2 deletion; ARS: aminoacyl t-RNA synthetase; CEA: carcinoembryonic antigen; $\mathrm{CI}$ : confidence interval; FBP: FUSE-binding protein; IHC: immunohistochemistry; ELISA: enzyme-linked immunosorbent assay; OS: overall survival; PFS: progression/recurrence-free survival; TGF: tumor-derived growth factor; TNF: tumor necrosis factor; TRAF2: TNF receptor-associated factor 2.

\section{Acknowledgement}

This study was supported by the Global Frontier National Research Foundation, Ministry of Science, ICT \& Future Planning (MSIP) of Korea 2014M3A6A4074817, NRF-M3A6A4-2010-0029785 and NRF-2015M3A6A4065724.

\section{Competing Interests}

The authors have declared that no competing interest exists.

\section{References}

1. Siegel R, Ma J, Zou Z, Jemal A. Cancer statistics, 2014. CA Cancer J Clin. 2014; 64: 9-29.

2. Choi JW Kim DG, Lee $\mathrm{AE}$ Kim HR, Lee JY, Kwon $\mathrm{NH}$, et al. Cancer-associated splicing variant of tumor suppressor AIMP2/p38: pathological implication in tumorigenesis. PLoS Genet. 2011; 7: e1001351.

3. Kim MJ, Park BJ, Kang YS, Kim HJ, Park JH, Kang JW, et al. Downregulation of FUSE-binding protein and c-myc by tRNA synthetase cofactor p38 is required for lung cell differentiation. Nat Genet. 2003; 34: 330-6.

4. Han JM, Park BJ, Park SG, Oh YS, Choi SJ, Lee SW, et al. AIMP2/p38, the scaffold for the multi-tRNA synthetase complex, responds to genotoxic stresses via p53. Proc Natl Acad Sci U S A. 2008; 105: 11206-11.

5. Choi JW, Kim DG, Park MC, Um JY, Han JM, Park SG, et al. AIMP2 promotes TNFalpha-dependent apoptosis via ubiquitin-mediated degradation of TRAF2. J Cell Sci. 2009; 122: 2710-5.

6. Chang SH, Chung YS, Hwang SK, Kwon JT, Minai-Tehrani A, Kim S, et al. Lentiviral vector-mediated shRNA against AIMP2-DX2 suppresses lung cancer cell growth through blocking glucose uptake. Molecules and cells. 2012; 33: 553-62.

7. Hwang SK, Chang SH, Minai-Tehrani A, Kim YS, Cho MH. Lentivirus-AIMP2-DX2 shRNA suppresses cell proliferation by regulating Akt1 signaling pathway in the lungs of AIMP2(+)/(-) mice. Journal of aerosol medicine and pulmonary drug delivery. 2013; 26: 165-73.

8. Yum MK, Kang JS, Lee AE, Jo YW, Seo JY, Kim HA, et al. AIMP2 Controls Intestinal Stem Cell Compartments and Tumorigenesis by Modulating Wnt/beta-Catenin Signaling. Cancer research. 2016; 76: 4559-68.

9. Choi JW, Lee JW, Kim JK, Jeon HK, Choi JJ, Kim DG, et al. Splicing variant of AIMP2 as an effective target against chemoresistant ovarian cancer. J Mol Cell Biol. 2012; 4: 164-73.

10. Lee HS, Kim DG, Oh YS, Kwon NH, Lee JY, Kim D, et al. Chemical suppression of an oncogenic splicing variant of AIMP2 induces tumour regression. The Biochemical journal. 2013; 454: 411-6.

11. Hsiao KC, Shih NY, Chu PY, Hung YM, Liao JY, Chou SW, et al. Anti-alpha-enolase is a prognostic marker in postoperative lung cancer patients. Oncotarget. 2015; 6: 35073-86.

12. Lui NS, Yang YW, van Zante A, Buchanan P, Jablons DM, Lemjabbar-Alaoui H. SULF2 Expression Is a Potential Diagnostic and Prognostic Marker in Lung Cancer. PloS one. 2016; 11: e0148911.

13. Xie P, Li X, Tan X, Sun X, Wang C, Yu J. Sequential Serum Let-7 Is a Novel Biomarker to Predict Accelerated Reproliferation During Fractional Radiotherapy in Lung Cancer. Clinical lung cancer. 2016; 17: e95-e101.

14. Stieber P, Hasholzner U, Bodenmuller H, Nagel D, Sunder-Plassmann L, Dienemann H, et al. CYFRA 21-1. A new marker in lung cancer. Cancer. 1993; 72: $707-13$.
15. Wieskopf B, Demangeat C, Purohit A, Stenger R, Gries P, Kreisman H, et al. Cyfra 21-1 as a biologic marker of non-small cell lung cancer. Evaluation of sensitivity, specificity, and prognostic role. Chest. 1995; 108: 163-9. 